practice prior to independent practice. Finally, a patient satisfaction questionnaire was developed.

Strategy for change Following the audit, we met with the consultant body and ED management, who approved our plans to introduce the ketamine sedation service.

Once the guideline was written, we liaised again with consultants and paediatric ED nurses to discuss the practicalities of the service being implemented. Here, we also developed the strategy for training of medical staff undertaking ketamine sedation.

The final pack of written guideline, SOP, competency assessment and patient questionnaire was submitted to ED consultants and management. Since approval we have commenced the sedation service within our ED

Measurement of improvement Since implementation of the guideline, we have sedated 4 children in the ED. Using the patient satisfaction questionnaire, feedback, has so far been positive. We will look to audit all children sedated in the ED in February 2015, assessing levels of adherence to the guideline, patient outcomes and patient satisfaction. Cost-Benefit analysis is also being undertaken at present.

Effects of changes Since our service commenced, we have avoided 4 patients needing to be put under GA, consequently increasing the efficiency of patient care, without affecting quality, and freeing up vital space on both the paediatric ward and operating theatre time.

Having adequate staffing levels to conduct sedations, along with space in the department, especially when the department is busy has proved difficult. Sedations are only conducted between 0800 and 2000, and in some cases, it will be necessary to bring children back the following morning to address this issue.

Lessons learnt Due to the small numbers of sedations being conducted, and the shift nature of Emergency Medicine work, our training has been limited to ad hoc experiential training and competency assessment of senior trainees. A comprehensive training programme, consisting of theoretical, simulation and experiential learning for all trainees at the commencement of the service may alleviate some anxieties amongst trainees and increase competency levels.

Message for others Drawing knowledge from trainee's previous experiences in other ED's where successful ketamine sedation service already exists, we were able to understand the pragmatic implications of introducing such a service. Discussion of ideas and methods of practice in different departments should be encouraged within the multidisciplinary team to promote improvement and development of services.

\section{G537(P) PROSPECTIVE RE-AUDIT OF CENTRAL LINE ASSOCIATED BLOODSTREAM INFECTIONS ON THE NEONATAL UNIT FOLLOWING GUIDELINE IMPLEMENTATION}

${ }^{1} \mathrm{C}$ Ratcliffe, ${ }^{2} \mathrm{~A}$ Abelian, ${ }^{3} \mathrm{R}$ Reynolds. ${ }^{1}$ Paediatrics, University Hospital of Wales, Cardiff, UK; ${ }^{2}$ Paediatrics, Wrexham Maelor Hospital, Wrexham, UK; ${ }^{3}$ GPVTS, Swansea, Swansea, UK

\subsection{6/archdischild-2015-308599.488}

Context This audit was carried out in Wrexham Maelor Hospital's (WMH) neonatal unit (NNU) and conducted by the authors (two FY2 doctors and Staff Grade paediatrician). The guideline which has been implemented was approved by both medical and nursing teams.

Problem A prospective audit performed in 2010-2011 showed that Wrexham Maelor Hospital's (WMH) neonatal unit (NNU) had a high rate of central-line associated bloodstream infections
(CLABSI) as compared to the rate reported by Centres for Disease Control and Prevention (USA). CLABSI have a high cost in terms of both morbidity and financial expenditure, and are preventable.

Assessment of problem and analysis of its causes To quantify the problem the infection rate was expressed as number of central lines days per one CLABSI. CLABSI was defined as growth of the same organism in blood and central line tip cultures (obtained within 48 hrs of each other). The causes of CLABSI were assessed using CDC recommendations (Guideline for the prevention of intravascular catheter-related infections, 2011, CDC, USA). To impose the changes, CDC recommendations were adapted, local guideline developed and medical and nursing staff educated.

Intervention A guideline was introduced in January 2013 that recommended use of $0.5 \%$ chlorhexidine in $70 \%$ alcohol (Hydrex ${ }^{\circledR}$ Pink), “check and do" list for clinicians, purchase of bundled supplies, continuous staff education, and nurse empowerment to stop non-urgent insertions if proper procedures were not followed.

Study design This was a prospective re-audit.

Strategy for change The "check-and-do" list was approved by the medical and nursing teams. The results of the initial audit were presented to the paediatric team along with the new guideline. It was agreed that the guideline would be followed and completed check and do list inserted into the notes of every patient who had a central line inserted from June 2013 onwards. The re-audit looked at the rate of CLABSI for all central lines (long lines, umbilical venous catheters [UVCs] and umbilical arterial catheters [UACs]) inserted between June 2013 and May 2014, and was prospective.

Measurement of improvement The results of these pre- and post-guideline audits were compared. The CLABSI rate in the pre-guideline audit was 10 in 179 long line days. In the re-audit, the CLABSI rate was 0 in 201 long line days, and 3 in 530 all central line days (long lines, UVCs and UACs; Note the preguideline audit only looked at infections associated with long lines). The difference between the distributions of gestational age in the audits was non-significant (Mann-Whitney test).

Effects of changes The re-audit data showed that the rate of CLABSI in the NNU have significantly decreased since the implementation of the guideline. CLABSI-associated morbidity and mortality have also decreased, which clearly benefited the patient group.

Lessons learnt It is helpful when implementing change and new guidance to work as a multi-disciplinary team. We found that by doing so, the new guidance was well received and adhered to.

Message for others We would recommend that all neonatal units establish an ongoing audit of CLABSI, and produce a guideline

\begin{tabular}{|c|c|c|c|}
\hline & 2010-2011 & 2013-2014 & $p$ \\
\hline Number of lines & 41 & 72 & $<0.001$ \\
\hline Total line days & 179 & 530 & $<0.001$ \\
\hline $\begin{array}{l}\text { Average time line in } \\
\text { situ }\end{array}$ & 4.37 days & 7.36 days & $<0.01$ \\
\hline Number of infections & 10 & 3 & $<0.001$ \\
\hline Average gestation & 30.19 weeks & 32.67 weeks & 0.054 \\
\hline Average weight & No data & $1897 \mathrm{~g}$ & \\
\hline
\end{tabular}


and "check and do" list if there is not already one in use. Our experience showed that significant reduction in CLABSI rate can be achieved in a relatively small NNU, over a relatively short time scale with similar effectiveness to that shown in larger NNUs and with close to zero additional costs.

\section{G538(P) REDUCING PRESCRIBING ERRORS BY INTRODUCING A PAEDIATRIC PRESCRIBING WEB-BASED LEARNING MODULE IN A TERTIARY CHILDREN'S HOSPITAL}

\author{
$1,{ }^{2} \mathrm{~V}$ Monnelly, ${ }^{2} \mathrm{~S}$ Kiff, ${ }^{2} \mathrm{R}$ Ardill. ${ }^{1}$ Neonatal Unit, Royal Infirmary of Edinburgh, Edinburgh, \\ UK; ${ }^{2}$ General Paediatrics, Royal Hospital for Sick Children, Edinburgh, UK
}

\subsection{6/archdischild-2015-308599.489}

Background Medication errors are a significant problem in theUK, and have the potential to cause harm. Children are particularly vulnerable. Errors occur at different decision-making points in the medicines pathway, and prescribing errors constitute a significant but potentially modifiable step in this process, which we targeted.

Our hospital regularly audits prescribing against our local prescribing policy (the gold standard), which is based upon GMC guidance for safe prescribing. The rate of prescription error was $67 \%$ in 2010 . We defined 'prescription error' as any deviation from the gold standard.

From August 2010, our mandatory junior doctor induction was modified to include a $2 \mathrm{~h}$ lecture on paediatric prescribing (delivered by pharmacist and doctor) and a paediatric prescribing test. This combination was shown to reduce prescribing errors from $67 \%$ to $52 \%$, and reduce incorrect or absent allergy information on prescription charts from $36 \%$ to $15 \%$ in 2010 and 2012 respectively.

The problem Although effective in improving prescribing, the lecture had a large volume of information to deliver in a limited time. Administering and marking the test was time consuming and providing individual feedback was logistically challenging.

A potential solution Web-based learning modules (WBLM) are accessible, low cost and can facilitate simultaneous education and assessment.

Aim To design and pilot a WBLM to replicate the previous content of prescribing induction. The long-term aim is to continue to improve paediatric prescribing within our hospital.
Primary objective Assess feasibility of introducing a WBLM as part of paediatric induction.

Secondary objective Assess the effect of the WBLM on prescribing practice.

The intervention The WBLM was designed by paediatricians (the authors) working with a Learnpro designer. It can be accessed remotely and is PC or tablet compatible.

There are 3 main sections. The first is an introduction to paediatric prescribing, revising pharmacokinetics and local policy for safe prescribing. The second section uses clinical cases to illustrate prescribing challenges and focuses on areas where errors frequently occur. Adverse drug reactions, antibiotic monitoring, medication error reporting and discharge prescriptions are covered. The final section focuses on intravenous fluids.

All sections contain links to relevant local clinical guidelines. Summative assessment questions are incorporated into the module, requiring trainees to obtain the correct answer before progressing.

Implementation The WBLM was designed in early 2014. Usability was tested in a small group prior to a pilot in August 2014 for junior medical and surgical doctors attending hospital induction.

Measurement of Improvement The Plan-Do-Study-Act cycle (PDSA) provides a structure for iterative testing of changes to improve quality. We are currently in the third cycle following the August 2014 pilot. All post-completion evaluations were positive. Minor technical issues reported have been addressed. The small scale testing has allowed adaptation according to feedback received.

All inpatient prescription charts, excluding PICU who use electronic prescribing, were audited in November 2014 (Figure 1).

There were 78 prescription charts containing 904 individual prescriptions. $85 \%$ of 'regular' prescriptions were prescribed correctly. Although only $68 \%$ of total prescriptions were correct, a significant proportion of errors were in 'once only' and 'PRN' sections, commonly completed in $\mathrm{A}+\mathrm{E}$ by doctors, who had not undertaken the WBLM. From 2015 the WBLM will be mandatory for all trainees rotating through the hospital, including $\mathrm{A}+\mathrm{E}$, anaesthesia and PICU.

Conclusion It is feasible to implement a WBLM as part of paediatric prescribing induction. Extending implementation to

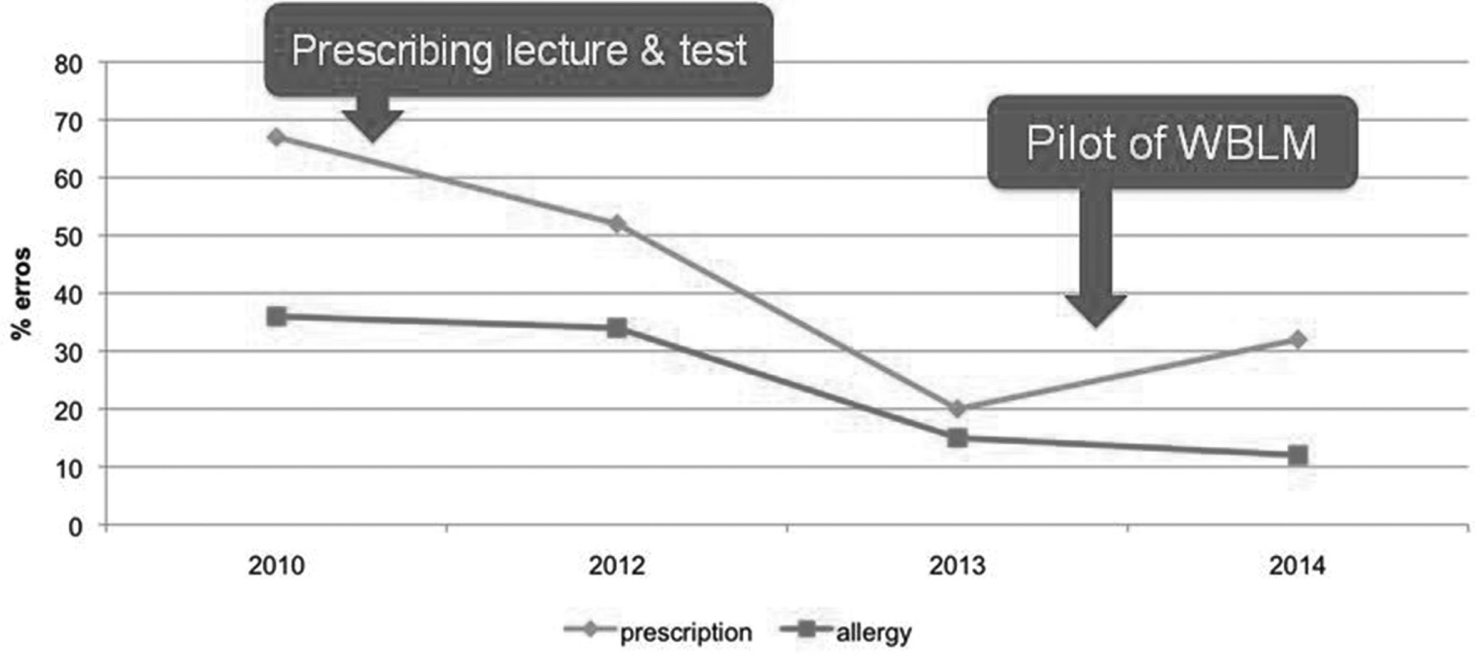

Abstract G538(P) Figure 1 Improving trends in prescription error and allergy documentation over time 